\title{
Notas para el conocimiento de la comunicación en las ONG en Portugal
}

\section{Observations on the understanding of communication in Portuguese NGOs}

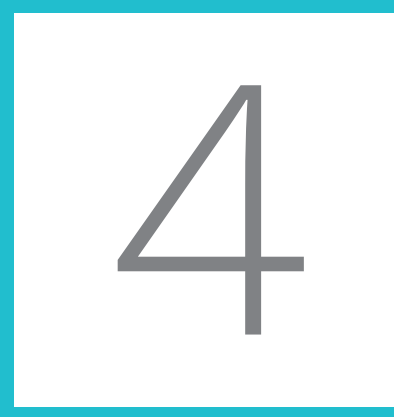

ARTÍCULO

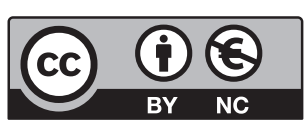

\section{Mafalda Eiró-Gomes}

Escola Superior de Comunicação Social Instituto Politécnico de Lisboa

PhD y Master en Ciencias de la Comunicación por la Universidade Nova de Lisboa (UNL). Profesora de Pragmática y Relaciones Públicas de la Escola Superior de Comunicação Social (ESCS-IPL). Consultora, pro, bono, de diversas organizaciones de la sociedad civil.

agomes@escs.ipl.pt

ORCID: 0000-0001-9573-5576

\section{César Pimentel Neto}

Escola Superior de Comunicação Social Instituto Politécnico de Lisboa

PhD en Ciencias de la Comunicación or el Instituto Universitario de Lisboa (ISCTE-IUL), Licenciado y Master en Relaciones Públicas por la Escola Superior de Comunicação Social (ESCS-IPL). El director de comunicaciones de la Plataforma Portuguesa das ONGD. Especialista en comunicación en el tercer sector y en comunicación en contexto deportivo. Profesor en la ESCS-IPL.

cneto@escs.ipl.pt

ORCID: 0000-0002-5542-6995 


\section{Cláudia Vasconcelos Silvestre}

Escola Superior de Comunicação Social Instituto Politécnico de Lisboa

PhD en Métodos Cuantitativos del Instituto Universitario de Lisboa (ISCTE-IUL), licenciado y master de Probabilidad y Estadística por la Facultad de Ciencias, Universidad de Lisboa (FCUL). Profesora en la Escola Superior de Comunicação Social (ESCS-IPL), donde coordina la Sección de Estadística.

csilvestre@escs.ipl.pt

ORCID: 0000-0002-8850-4304

Fecha de recepción: 30 de noviembre de 2017 / 19 de marzo de 2018

\section{RESUMEN}

Investigación con carácter exploratorio que permite un primer diagnóstico acerca de la importancia que las organizaciones no gubernamentales para el desarrollo (ONGD) dan a la comunicación. Si al principio, y por el número y diversidad de soportes de comunicación listados parece que la importancia atribuida a la comunicación, entendida aquí como constitutiva de las prácticas organizacionales, era una realidad, se constató sin embargo, que tal no es el caso en absoluto. La comunicación es, en la mayor parte de estas organizaciones, entendida como claramente instrumental y solamente pensada en su aspecto meramente discursivo.

\section{PALABRAS CLAVES}

Comunicación, estrategia, organizaciones no gubernamentales para el desarrollo, investigación, soportes de comunicación.

\section{Abstract}

In this exploratory research we aim to outline the role and importance that Portuguese non-governmental development organisations attach to the "communication" field. At first sight, considering the number of communication instruments in use, it might seem that this is a priority issue for organisations, but, in reality, this is not the case. In general, "communication" is considered instrumental and considered merely in terms of the desired discourse message in each case.

\section{KEYWORDS}

Communication, strategy, non-governmental organisations for development, research, instruments of communication

\section{INTRODUCIÓN}

Muniz Sodré (2014) comienza su último trabajo reiterando la ambigüedad de uno de los más comúnmente términos usados y de mayor penetración en todo nuestro espacio público: el concepto de comunicación. Desde el inicio de la segunda mitad del siglo XX lo encontramos en todas las materias - de la biología a la sociología, de la economía a la ecología o a la 
arquitectura - para referir una multiplicidad de situaciones, procesos o estructuras. Sin embargo, es en el lenguaje corriente de nuestra contemporaneidad que nos enfrentamos a su uso en una mayor multiplicidad de situaciones y a sus acepciones menos claras.

Ciertamente que la dificultad que esta área disciplinaria enfrenta en el día a día organizacional tiene relación con su proximidad en cuanto a la designación, de algo que parece inherente a la especie humana, "todos comunicamos", y con las numerosas designaciones que sus profesionales son contratados en el mercado portugués, ya sea como: "técnico de comunicación", "asesor o consultor en relaciones públicas", "especialista en las relaciones con los medios de comunicación", "agente de información", solo para enumerar una pequeña parte de los títulos que encontramos tanto en el sector privado como en el público, o el que nos interesa aquí, el designado generalmente como tercer sector.

En este artículo, que es el resultado de una investigación con carácter exploratorio, que consiste en una revisión de monografías de maestría sobre el tema en cuestión, entre 2008 y 2015, y del trabajo de investigación empírica, con una primera parte que consta de entrevistas informales y una segunda parte esencialmente cuantitativa tanto en la recogida como en análisis de datos, desarrollada durante los meses de junio y julio de 2016 con las organizaciones no gubernamentales para el desarrollo (ONGD) en Portugal, se hace un primer diagnóstico acerca de la importancia que se da a la "comunicación" en estas organizaciones. Del universo de 185 ONGD registradas en el Ministerio de Asuntos Exteriores en mayo de 2016, y habiendo sido efectuados al menos tres intentos de contacto telefónico, después de un primer contacto por correo electrónico, se obtuvo un universo de encuestados de 77 organizaciones, de las cuales 42 pertenecen a la Plataforma Portuguesa de las ONGD.

Tanto en el transcurso de la revisión hecha a los trabajos realizados en el área de comunicación en las organizaciones de la sociedad civil - en el contexto de la maestría en Gestión Estratégica de Relaciones Públicas - y guiados por uno de los investigadores que suscribe este trabajo, como en el transcurso del trabajo como técnico de comunicación específicamente en el área de organizaciones no gubernamentales de otro investigador llegó a estar claro que, aunque sin que lo pudiéramos asegurar de forma categórica y justificada, el área de la comunicación es un pariente pobre y claramente prescindible en este sector, ya sea por cuestiones de una gestión muy dependiente de la financiación a corto plazo o, lo que parece más plausible, una total ignorancia de su verdadero papel.

\section{LA COMUNICACIÓN ESTRATÉGICA EN EL TERCER SECTOR}

Es en el seno de la sociedad civil que los movimientos sociales se vuelven organizados, se constituyen como grupos en defensa de un interés en particular, o causa, ganan voz contribuyendo a denunciar situaciones de injusticia social o ambiental, violaciones de derechos humanos o simplemente respondiendo a las necesidades de las comunidades locales apoyando a la tercera edad o a la infancia.

Las Organizaciones de la Sociedad Civil (OSC) han aumentado en número y tamaño, en las últimas décadas, y ahora no sólo son importantes agentes sociales sino que también lo son a nivel económico - a medida que atraen más financiación externa y consolidan el papel como socios preferenciales de muchos gobiernos y supra-entidades, ganando más visibilidad y al 
mismo tiempo ganando protagonismo en la formulación de políticas, en la cobertura de los medios y en interés del público (Riddell y Bebbington, 2013). La forma como designamos a los grupos que surgen dentro de nuestras sociedades y que grosso modo podemos caracterizar como pertenecientes al sector social o al tercer sector, parece depender no sólo de propuestas de entidades supranacionales, sino esencialmente de los marcos jurídicos de los distintos países donde están inscritos. Si bien es cierto que no siempre encontramos consenso sobre la designación que adoptar para referirnos a estas organizaciones, también por algunos autores señaladas como organizaciones no gubernamentales $u$ organizaciones sin fines de lucro, lo que parece sí consensuado es lo que están haciendo para promover el bien público fomentando la participación y el empoderamiento de los ciudadanos (Blair, 2013; UNDP, 2012; Mishra, 2012).

\subsection{ONGD}

En Portugal, las organizaciones que pueden considerarse como pertenecientes a este grupo, muy heterogéneo tanto desde el punto de vista de su constitución como de sus objetivos, son casi tan antiguas como el país, especialmente las de naturaleza confesional $y$, en este caso, pertenecientes o dependientes de la Iglesia Católica Romana. La creación de estas organizaciones a menudo se confunde con los inicios de la nacionalidad, después de haber dominado el ámbito caritativo, en su significado real, hasta prácticamente el final del siglo XX.

Los cambios a nivel internacional, ya visibles desde el final de la Segunda Guerra mundial, y el surgimiento de la democracia en Portugal en la década del 70 del siglo pasado cambiaron el panorama del tercer sector, también por muchos señalado como sector social en Portugal, llevando a una creciente profesionalización del mismo. Las Organizaciones de este sector más allá de su rol como interlocutores privilegiados de los gobiernos y entidades supra en la discusión de asuntos de interés público, como entrenadores y activistas de la opinión pública, se han convertido en las últimas tres décadas en elementos a tener en cuenta al mismo tiempo como empleadores de jóvenes licenciados en los campos de la sociología, biología, trabajo social, las relaciones internacionales, y también en el campo de la comunicación, en sus diversas vertientes.

Estamos interesados en caracterizar aquí un pequeño grupo entre la inmensa diversidad de grupos que existen en el espacio entre lo público y lo claramente individual, las designadas como ONGD. Éstas se rigen, en Portugal, por la ley n 66/98 del 14 de octubre de 1998, que aprueba el estatuto de organizaciones no gubernamentales de cooperación para el desarrollo, definiéndolas como "personas colectivas de derecho privado, sin fines de lucro" ( $p$. 5308). Sus objetivos son el diseño y la ejecución de programas y proyectos de cariz social, cultural, ambiental, cívico y económico en países en vías de desarrollo. Se consideran como grandes áreas de actuación la Cooperación para el Desarrollo, la Ayuda Humanitaria, la Ayuda de Emergencia y la promoción y protección de los Derechos Humanos. Pueden adoptar diferentes formas jurídicas como las de asociación, fundación, cooperativa u organización erigida canónicamente, siendo que todas deben estar registradas en el Ministerio de Relaciones Exteriores, entidad responsable de reconocer su estatuto.

\subsection{COMUNICACIÓN EN LAS ONGD}

Los conceptos de comunicación institucional, comunicación corporativa y relaciones públicas son de acuerdo a algunos autores conceptos 
sinónimos, de acuerdo otros, no tanto. No entraremos aquí en esa discusión, aceptando que para efectos de este artículo esa diferencia no es demasiado relevante (para una discusión sobre estos conceptos ver Nunes y Eiró-Gomes, 2013) y optando en términos generales por comunicación institucional o comunicación sin otras especificaciones.

Sin embargo, no podemos prescindir de aclarar que la noción de Relaciones Públicas debe ser entendida teniendo en cuenta la acepción propuesta por White y Mazur (1995), como una función que gestiona las relaciones entre los diferentes públicos, entre éstos y las organizaciones, en una perspectiva de adaptación mutua y desarrollo de intereses comunes. De alguna manera lo que aquí defendemos es una visión de las Relaciones Públicas cuando éstas se entienden como fundamentales en la creación de relaciones con la comunidad, un modelo de Relaciones Públicas centrado en una noción de compromiso, es decir, cuando además de las relaciones de intercambio, crean relaciones que pueden beneficiar los públicos sin necesidad de beneficiar a la organización. Éste es un modelo ya descrito por Grunig (1997) y que molda gran parte de lo que hoy ha sido designado como la teoría crítica en las Relaciones Públicas (Coombs y Holladay, 2014; Eiró-Gomes y Lourenço, 2009; Eiró-Gomes, 2005, 2006; Nunes Y Eiró-Gomes, 2013).

Aunque a menudo es vista como un área autónoma, o desde un marco de lo que es para muchos designado como "comunicación para el cambio social y de comportamiento", a raíz de la Johns Hopkins Public Health School, la Comunicación para el Desarrollo es actualmente una de las funciones de las Relaciones Públicas (o de la Comunicación Institucional) y su importancia dentro de las organizaciones del tercer sector es incuestionable (Broom y Sha 2013; Wilcox, Cameron y Xifra 2012; Eiró-Gomes y
Nunes, 2012). Algunos autores señalan también la importancia de la "comunicación para el desarrollo" en la construcción de la identidad de la organización, así como en su proyección junto a las comunidades con las cuales tejen redes de cooperación y se maximizan los pocos recursos existentes en pro de cambios sociales beneficiosos para las comunidades involucradas (Patterson y Radtke, 2009).

Sin embargo sabemos que, al contrario de lo que defiende la especialidad de la literatura (Patterson y Radtke, 2009), en muchas organizaciones del tercer sector se encuentran grandes disparidades en comparación con lo que consideran pertenecer o no al área de comunicación institucional (Relaciones Públicas) y lo que consideran como siendo parte de lo que designan como comunicación para el desarrollo. Si en este tema podemos tener algunas interrogaciones, lo mismo no es posible en las áreas que, éstas sí, no presentan ninguna disensión teórica, pues son relativas a las funciones generales consideradas en cualquier organización como pertenecientes al área de Relaciones Públicas, tales como: Comunicación Interna/Relaciones con los empleados; Gestión de Asuntos; Gestión de Comunicación de Riesgos y Crisis; Gestión de Eventos; Relaciones con la Comunidad; Relaciones con los Inversores; Relaciones con el Gobierno y Asuntos Públicos (grupos de interés); Relaciones con los Medios de Comunicación Social; Responsabilidad Social de las empresas /Recaudación de fondos; patrocinios y promoción.

Creemos que en las ONG la comunicación será exigida que sea no solamente guardián de los valores, la marca y la reputación de la organización, sino que también el elemento clave para el desarrollo de capacidades (empowerment) de los individuos, con el fin de llevarlos a actuar (advocacy), y por lo tanto convertirse en parte activa en la toma de decisiones, tanto en la or- 
ganización como, esencialmente, dentro de sus comunidades, para el bien común (Eiró-Gomes y Lourenço, 2009).

\section{DISEÑO DE LA INVESTIGACIÓN}

Ciertamente, la experiencia personal de los investigadores, sus intereses y opciones teóricas afectan la forma de llevar a cabo una investigación bien como el objeto en estudio. Por lo tanto, consideramos que este trabajo se basa claramente en una visión del tipo preconizado por la escuela pragmatista del trabajo de investigación, donde la relación entre el mundo académico y las necesidades de la sociedad en la que operamos es una constante (Creswell, 2014). Buscamos al mismo tiempo satisfacer la necesidad de la Plataforma Portuguesa de ONGD y la necesidad de realizar una reflexión teórica sobre las prácticas de comunicación en un sector en crecimiento, lo que representa, por tanto, uno de los objetivos de las obras que forman parte de un paradigma pragmático, es decir, una investigación centrada en un problema real y los resultados tendrán consecuencias directas en la re-ecuación de ese mismo problema. Para un análisis más exhaustivo de nuestro tema, y dada la falta de información existente sistemática y la heterogeneidad de nuestro tema, optamos por el uso de diferentes formas de recopilar y analizar los datos, generalmente designado desde el punto de vista metodológico como el uso de la convergencia de métodos mixtos paralelos (Berger, 2014; Creswell, 2014).

Así, se estableció como primer objetivo de esta investigación, entender cuál es la percepción de cinco profesionales de la comunicación, con formación en el área de la comunicación estratégica a nivel de licenciatura o maestría, trabajando en organizaciones no gubernamentales, del posicionamiento de sus instituciones frente a los dos temas a saber:

- a) Si la comunicación es considerada por las organizaciones como una vertiente estratégica u operativa; y

- b) si la comunicación es transversal a todos los departamentos de la organización o simplemente se limita a ciertos aspectos "institucionales".

Las entrevistas informales con carácter exploratorio tuvieron lugar en mayo de 2016 y permitieron, al mismo tiempo con el análisis del contenido de cariz cualitativo realizado a las disertaciones, informes o proyectos de maestría ya referidos, percibir que los puntos de vistas presentados en las ONG apuntan claramente a una comprensión aún muy rudimentaria de la función "comunicación" en estas organizaciones.

La distinción entre lo que puede ser el campo de los "comunicadores", la comunicación institucional y todo el "trabajo" de "comunicación" realizado por estas organizaciones en el área de "comunicación para el desarrollo", no es en absoluto visto como "comunicación", sino que es visto como educación o cualquier otra designación dependiendo de que si el trabajo desarrollado se sitúa más en el área de salud, de finanzas, de agricultura o de alfabetización, se vuelve evidente y hasta esperado por nosotros, sorprendiéndonos el papel meramente instrumental que todavía se atribuye a la "comunicación".

Es interesante que incluso en instituciones que cuentan en sus estructuras organizativas con un departamento, dirección o sección de comunicación (bajo múltiples nombres), este órgano tiene una función puramente técnica, por lo que no aporta ningún valor añadido estratégico y es únicamente responsable de los aspectos de redacción o gráficos de diversos 
instrumentos; actualizaciones de canales digitales existentes - página web, facebook, boletines de noticias - y producción de piezas de comunicación con naturaleza suelta y en estricta dependencia de otros departamentos.

Dado que tienen visiones muy discursivas e instrumentales de la "comunicación", el área de medios de comunicación es la que presenta una mayor inversión por parte de las organizaciones (también porque a menudo estos soportes son el resultado de las demandas hechas en los proyectos financiados por entidades externas). Sin embargo, aunque en gran número y con unos gráficos de calidad, vemos que los medios de comunicación no están dirigidos a un público específico o áreas estratégicas de la organización, y que en el fondo, sólo existen para que la organización pueda "decir" que se comunica (Cano, 2014; Machado, 2009; Montenegro, 2012; Nunes, 2011; Pereira, 2015; Santos, 2015; Sousa, 2013).

Con base en la información obtenida en la primera fase de la investigación decidimos reformular lo que pensamos inicialmente como nuestro trabajo de investigación cuantitativo y, si lo desean, comenzar por los aspectos claramente más básicos y el marco estructural, es decir, qué aspectos referentes a la comunicación hay en toda una gama de organizaciones que se nos presentan bajo una misma designación y que legalmente constituyen una categoría en el seno de las OSC, y cuál es la atención prestada a cuestiones designadas a menudo como educación o información.

Uno de los aspectos que también buscamos examinar fue la posición del profesional responsable de la comunicación en la institución, su formación académica, bien como la existencia o no de un departamento o sección autónomo responsable por la comunicación.
Teniendo en cuenta los datos recogidos, tanto en la revisión bibliográfica específica como las entrevistas exploratorias, todas las cuestiones de carácter no operativo se han dejado para un futuro estudio cualitativo, debido a la necesidad de mapear de manera sistemática lo que nos dijeron que existía, antes de entrar en aspectos conceptuales de un nivel diferente.

\subsection{ENCUESTA}

Las ONG que no respondieron al correo electrónico y en dicho contacto se coordinó la fecha de aplicación del cuestionario que fue a su vez remitido por e-mail. Todas las organizaciones con las que no era posible hablar después de tres intentos de contacto telefónico, en un periodo de quince días, y después de reenviar la carta una vez más por e-mail se las consideró no respondedoras (43). Cuando las organizaciones, a pesar de que era posible establecer el contacto, no se negaron a responder de inmediato al cuestionario (por correo electrónico o contacto telefónico) se las volvió a contactar, hasta tres veces, para programar la aplicación del cuestionario; después del tercer intento la organización era considerada como parte de las que se negaban a contestar (47). Finalmente, encontramos 19 organizaciones sin ningún tipo de información relacionada a números de teléfono u otros contactos válidos. Por lo que pudimos observar, hay algunas organizaciones que a pesar de que constan en las listas oficiales no tienen existencia real. De acuerdo con algunas escuelas y paradigmas de comunicación cualquier comportamiento, o no comportamiento, es un acto comunicativo, pero no abordaremos aquí cualquier tipo de especulaciones sobre aquellas que no mostraron interés en participar en el estudio. En un intento de promover una mayor receptividad por parte de los diferentes actores, el grupo de investigación 
contó con el apoyo de la Plataforma Portuguesa de ONGD en la difusión de la iniciativa.

El cuestionario, meramente de carácter descriptivo, se dividió en tres grandes áreas que corresponden a los tres objetivos de investigación que guiaron esta fase del estudio:

- a) Entender la importancia que estas organizaciones atribuyen a la expresión de su identidad (lo que algunos autores designan como marca, es decir, una identidad visual conjunta, y de la única proposición de venta que en este contexto podemos considerar como la unión de la visión, los valores y la misión);

- b) Comprender cuál es la importancia asignada a los diferentes soportes de comunicación, con especial interés en lo relacionado a los medios de comunicación / medios digitales (redes sociales y Web);

- c) Caracterizar quién es responsable de las áreas consideradas de manera inequívoca desde un punto de vista teórico como perteneciente a un profesional de la comunicación y que, en general, son parte de las OSC.

\subsubsection{ANÁLISIS E INTERPRETACIÓN DE DATOS}

El análisis y la interpretación de los datos están organizados en función de los tres objetivos de investigación mencionados anteriormente. El análisis tiene una vertiente descriptiva y también interpretativa. Fueron considerados como aspectos relevantes:

\section{- a) Importancia dada a la expresión de su identidad y a los diferentes soportes.}

Al analizar los datos recogidos a través del cuestionario, se puede concluir que las organizaciones conceden una gran importancia a la expresión de su identidad, y más del 90\% de las organizaciones encuestadas ponen el logo en los medios de comunicación que tienen, la única excepción sucede con la revista (75\%), pero en este caso se obtuvieron 3 respuestas positivas de 4 (ver tabla 1).

Se pudo constatar también que esta preocupación está igualmente presente en los soportes Administrativo y logísticos, más del $80 \%$ de las ONG ha puesto su logo en los medios de comunicación que utilizan, con la excepción de los coches, como puede verse en la tabla 2.

Se constató que, en general, ya sea por problemas de comunicación o por la imposición de los financiadores, las organizaciones entienden la importancia de la expresión de su identidad, de la comunicación de su marca en todos los medios de comunicación y soportes administrativos / logísticos que utilizan.

Con este cuestionario también fue posible enumerar los medios de comunicación utilizados por las ONG portuguesas (ver tabla 1) y es de destacar el enfoque en la comunicación en el ámbito digital, más del 95\% de las organizaciones encuestadas tienen página web, página de Facebook y utilizan el correo electrónico.

Como complemento al cuestionario, en donde 74 ONGD encuestadas afirmaron que tenían página web, optamos por confirmar esta información mediante un análisis de los sitios web, con el objetivo principal de confirmar su existencia.

Esta verificación permitió constatar que de las 77 ONG encuestadas, 6 no tienen página web (y de estas 6, 3 habían afirmado tenerla), y otras 7 ONG tienen presencia en línea, pero la apuesta pasa por un blog, páginas que tienen apenas la descripción de la ONG, los contactos y sitios web, sin informaciones básicas acerca de la organización, tales como la misión, descripción o los proyectos y sin ningún cuidado a nivel de diseño. 
Tabla 1. Los medios de comunicación utilizados por las ONG

\begin{tabular}{|c|c|c|c|}
\hline Soportes de comunicación & & Con el logotipo & Tiene $\%$ con el logotipo \\
\hline Sitio web & 74 & 72 & 97,3 \\
\hline Facebook & 73 & 68 & 93,2 \\
\hline Twitter & 27 & 27 & 100 \\
\hline Linkedln & 22 & 21 & 95,5 \\
\hline Correo electrónico & 75 & 68 & 90,7 \\
\hline Shop línea & 19 & 19 & 100 \\
\hline Roll Up & 60 & 58 & 96,7 \\
\hline Nota de prensa & 61 & 55 & 90,2 \\
\hline Revista & 15 & 15 & 100 \\
\hline Boletín & 21 & 21 & 100 \\
\hline Newsletter & 52 & 46 & 88,5 \\
\hline $\begin{array}{l}\text { Ediciones especiales (por ejemplo, el libro) conmemorativo, } \\
\text { etc.). }\end{array}$ & 38 & 37 & 97,4 \\
\hline Antecedentes de información del kit & 25 & 25 & 100 \\
\hline Carteles & 59 & 56 & 94,9 \\
\hline Videos & 62 & 60 & 96,8 \\
\hline De informe & 73 & 67 & 91,8 \\
\hline Plan de negocio & 75 & 69 & 92 \\
\hline Informe del Proyecto & 70 & 64 & 91,4 \\
\hline Aplicación formas & 54 & 51 & 94,4 \\
\hline Descripción del proyecto & 62 & 58 & 93,5 \\
\hline De la Letra & 63 & 59 & 93,7 \\
\hline Sobre & 57 & 53 & 93 \\
\hline Tarjetas & 57 & 52 & 91,2 \\
\hline Folletos & 67 & 64 & 95,5 \\
\hline Oficial & 4 & 3 & 75 \\
\hline Pegatinas & 30 & 30 & 100 \\
\hline Regalos & 35 & 35 & 100 \\
\hline Juegos & 16 & 16 & 100 \\
\hline Bolsas & 31 & 30 & 96,8 \\
\hline
\end{tabular}

Parece que, a pesar de la creciente preocupación por los nuevos medios digitales, hay algunos defectos a este nivel y será importante, en una etapa posterior de esta investigación, realizar un análisis más exhaustivo de los contenidos de los sitios de las ONGD.

\section{b) Responsable de Comunicación}

Analizando el trabajo de comunicación de las ONG, se puede concluir que de las 77 organizaciones que respondieron, 26 no tienen departamento de comunicación, no tienen en su equipo ningún técnico que desempeñe estas funciones y tampoco trabajan con agencias. 
Tabla 2. Apoyo administrativo y logístico utilizado por ONG

\begin{tabular}{|c|c|c|c|}
\hline De apoyo administrativo y de logística se & & Con el logotipo & Ha \% con el logotipo de \\
\hline La fachada del edificio & 39 & 39 & 100 \\
\hline Sucursales fuera de Portugal & 14 & 12 & 85,7 \\
\hline Identificación de la sede de la puerta (zumbadores) & 45 & 42 & 93,3 \\
\hline Recepción & 41 & 34 & 82,9 \\
\hline Coches & 37 & 28 & 75,7 \\
\hline Banderas & 27 & 26 & 96,3 \\
\hline Carpas / Pabellones & 12 & 10 & 83,3 \\
\hline $\begin{array}{c}\text { Identificación de personas (chalecos) uniformes, } \\
\text { camisetas, etc.) }\end{array}$ & 46 & 45 & 97,8 \\
\hline Papelería & 68 & 67 & 98,5 \\
\hline sobres & 59 & 57 & 96,6 \\
\hline Presentación Tarjetas / identificación & 65 & 64 & 98,5 \\
\hline Otros dispositivos & 7 & 7 & 100 \\
\hline
\end{tabular}

Con relación a las 51 restantes, se verificó que 37 trabajan con técnicos especializados, 38 de ellas cuentan con un Departamento de Comunicación y 32 de ellas trabajan con Agencias, y 18 apuestan por una combinación de un departamento con reclutamiento de técnicos especializados y con los servicios prestados por una Agencia (Figura 1).

Cabe mencionar que las ONGD que tienen departamento utilizan diferentes nombres como el Dep. de comunicación y sostenibilidad, Dep de. Comunicación, Dep. de Comunicación y Desarrollo, Dep. Comunicación y Movilización, Dep. Información Pública, Dep. de Relaciones Públicas Dep. de Imagen y Comunicación y Dep. de Comunicación y Promoción.

Sin embargo, aunque parece que existe una apuesta por la comunicación, al analizar la formación del Responsable de Comunicación de estas ONG, sólo 19 de 51 tienen formación en Comunicación Organizacional y Relaciones Públicas, esto nos permite comprender que, aunque se da importancia a la comunicación, sigue siendo el pariente pobre y, para las ONG, per- sonas con formación en otras áreas pueden realizar esta función.

Todo nos lleva a pensar que las ONG no consideran esencial que el Responsable de Comunicación esté formado en comunicación y que éste debe tener una función puramente técnica, como se puede comprobar al analizar las responsabilidades a nivel de la comunicación en las organizaciones que se plantean en sus estructuras organizativas un departamento, dirección o sección de comunicación (Figura 2).

A pesar de la creciente preocupación por la comunicación, cuestionando a las ONG sobre las Campañas Institucionales, la Recaudación de Fondos (hay que señalar que en 5 ONGD la responsabilidad es exclusiva del Departamento de Recaudación de Fondos), las Relaciones con los Medios de Comunicación o la Comunicación de Proyectos dentro y fuera de Portugal, se verificó que en menos de la mitad de las organizaciones la responsabilidad recae sobre el técnico, departamento o agencia de comunicación y / o Relaciones Públicas, e incluso en 
Figura 1. Departamentos, técnicos y Agencias de comunicación en las ONG

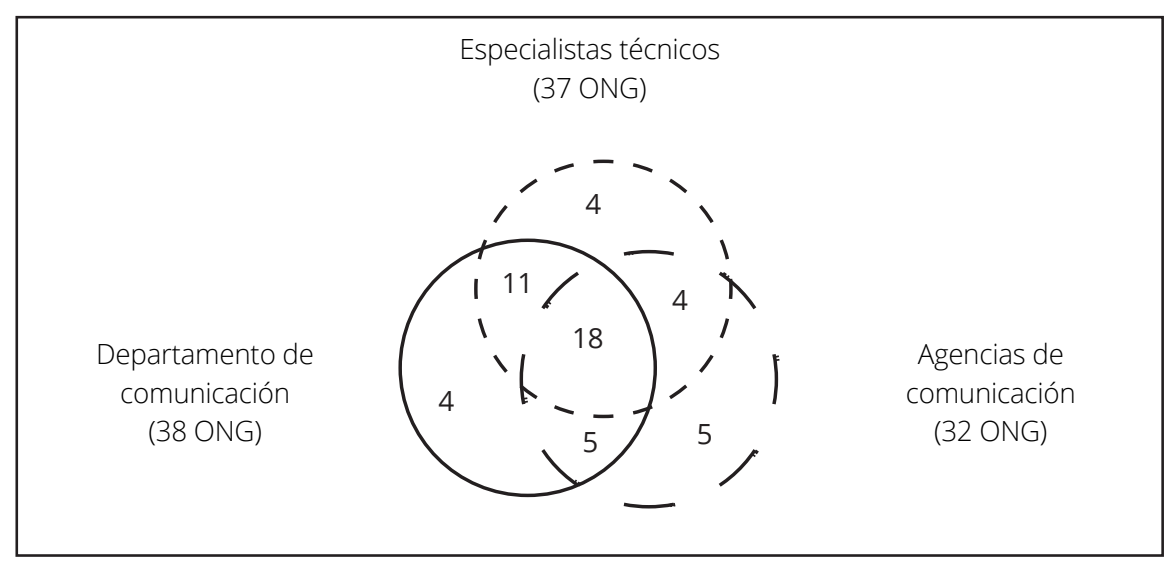

Figura 2. Responsabilidades del Responsable/Departamento/Agencia de Comunicación

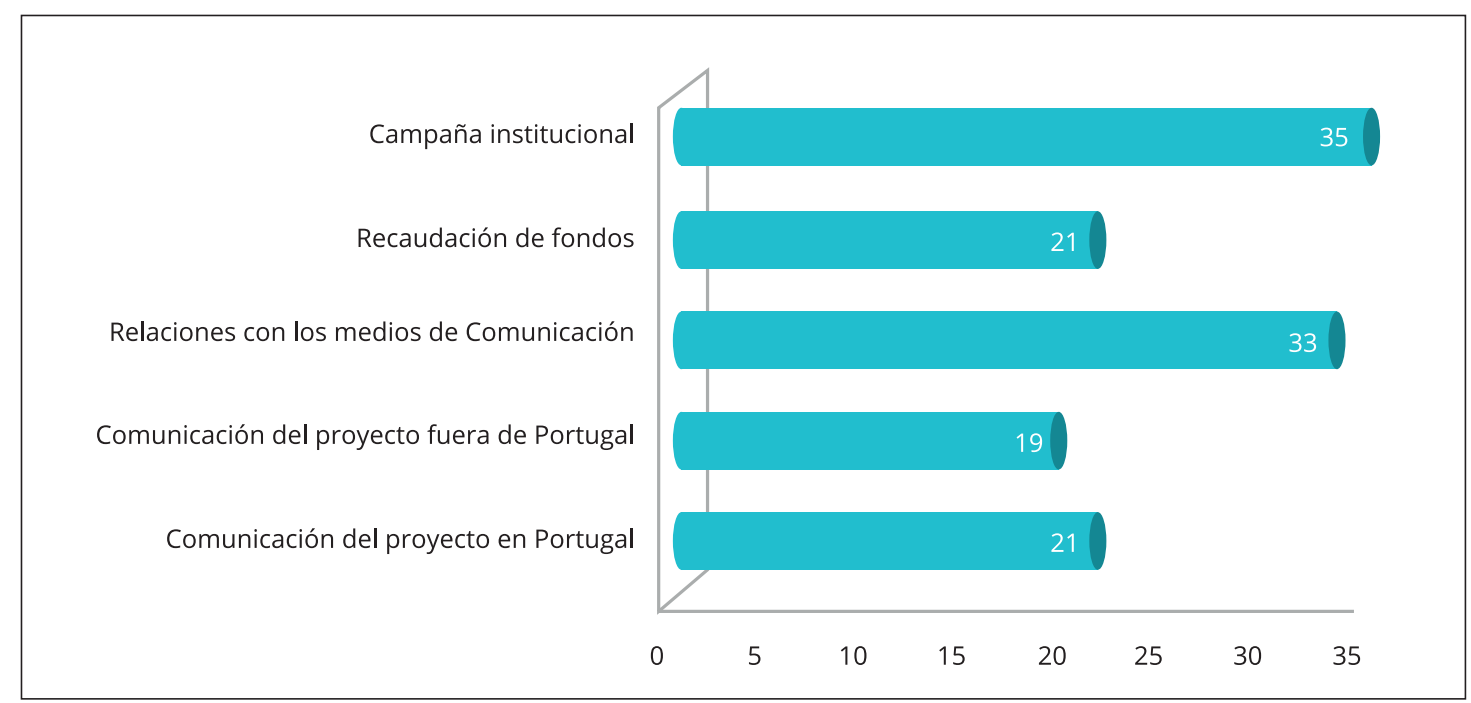

algunos casos de los casos presentados en el gráfico, la responsabilidad es compartida con la Dirección u otros departamentos de las organizaciones. Cabe resaltar que en muchos casos la responsabilidad de la gestión de la comunicación compete a la Junta, el Comité Ejecutivo, Departamento o Coordinador/a de Proyectos, sólo para ilustrar algunos ejemplos.

Esta realidad ayuda a confirmar que, a pesar de la creciente apuesta por la "comunicación" sobre todo en el número y la diversidad de medios/soportes de comunicación y el reconocimiento de la importancia de la comunicación para las ONG, el desconocimiento y la confusión del papel de los profesionales de la comunicación sigue siendo una realidad. La "comunicación" es aún vista desde una perspectiva meramente operacional, como la difusión de las actividades y proyectos, y no se mira a la Comunicación y a los profesionales de esta área bajo una perspectiva estratégica o de los órganos del personal. 


\section{CONCLUSIONES}

Se puede defender que en las organizaciones en general, la importancia que se les da a los profesionales de relaciones públicas o de comunicación suele ser inversamente proporcional a la importancia que supuestamente se atribuye a la comunicación. Este trabajo permite hacer notar que las organizaciones de la sociedad civil en Portugal, específicamente las ONG para el desarrollo, no son en absoluto una excepción. En muchas de estas organizaciones encontramos una gran ignorancia e incomprensión no sólo sobre las cuestiones conceptuales, sino también sobre cuál es el papel y cuáles son las competencias específicas de los profesionales de comunicación. La multiplicidad de contextos y situaciones donde las diferentes áreas que se definen aquí como "comunicación aplicada" son consideradas como un lujo, una banalidad o simplemente competencia de otros profesionales y no precisamente profesionales de comunicación, es una realidad constante.

Si bien es cierto que hay algunos trabajos genéricos que sugieren algunos pasos básicos para mejorar el trabajo en términos de Comunicación Institucional o de Relaciones Públicas en organizaciones de la sociedad civil (Patterson y Radtke, 2009), la realidad es que cada caso es diferente, tanto en lo que respecta a la calificación estructural y funcional de las organizaciones, como en el papel que se asigna a la "comunicación" en la estructura de esas mismas instituciones. El estudio desarrollado permite resaltar, como elemento presente en todas las organizaciones, la importancia que las instituciones investigadas tienden a asignar a los elementos que expresan su identidad, con énfasis en los aspectos gráficos, la visión y la misión, así como la importancia que sienten de estar presentes en la web, ya sea a través de sitios institucionales o bien a través de una presencia más o menos constante en las redes sociales. Cabe señalar también la importancia que atribuyen a diferentes aspectos de la vida organizacional, que siendo competencia específica de los profesionales de comunicación, éstas son asignadas a técnicos con formación en áreas tan dispares como la economía, la sociología o las relaciones internacionales.

También aquí, como en tantos otros estudios inspirados en la escuela pragmatista encontramos elementos que no fueron tomados en suficiente consideración porque no fueron objeto directo de nuestra investigación, pero aun así dichos elementos la condicionaron. Tal vez que lo que más condicionó esta investigación haya sido la multiplicidad de realidades, contextos, dimensión y prácticas organizacionales que encontramos en las 77 organizaciones estudiadas. Aunque aparentemente son parte de un mismo grupo, de una misma realidad, que permiten que en ámbitos como el económico o el sociológico se entiendan como pertenecientes a una misma categoría, en el transcurso de este trabajo nos encontramos ante una diversidad tal de organizaciones que, además de 
su fondo legal, muy poco tienen en común. En realidad, entre muchas de las ONGD estudiadas, no hay nada en común o similar en su estructura general, en el número de empleados, en la forma como trabajan o en la forma como entienden el papel que les incumbe desempeñar en la sociedad. Todas estas cuestiones condicionarán con seguridad la forma como entienden el trabajo de los profesionales de comunicación, dificultando también que éste pueda ser caracterizado con base en un cuestionario formal por lo cual la continuación de esta investigación debe ser llevada a cabo con entrevistas semi-estructuradas a las 77 organizaciones que respondieran a nuestro desafío.
"Si la comunicación es tan importante, ¿por qué no recibimos la atención que merecemos?" (Daly, 1998) parece una buena pregunta para concluir este trabajo. Hoy, tal como a finales del siglo pasado cuando dicha interrogación sirvió de titular para el artículo sobre el que nos referimos, el desconocimiento sobre el papel que los profesionales de comunicación desempeñan en las organizaciones, así como las necesidades específicas y los complejos técnicos de su labor parecen mantenerse. La comunicación, sin embargo, es considerada como importante.

Artículo escrito en el ámbito del proyecto "Comunicación en Organizaciones de la Sociedad Civil: del reconocimiento al conocimiento" apoyado por el Instituto Politécnico de Lisboa - IDI\&CA - IPL/2016/COSC-CR_ESCS y por la Plataforma Portuguesa das ONGD.Los autores agradecen a todas las organizaciones no gubernamentales para el desarrollo que contestaron al cuestionario, a los alumnos Inês Castelo, Pedro Rondão, Tânia Amaral y Vlada Domanska de la licenciatura de Relaciones Públicas y Comunicación Empresarial (2014-2017) de la ESCS - Escola Superior de Comunicação Social, Instituto Politécnico de Lisboa su apoyo en la recogida de datos y a la Maestra Sandra Cárdenas el apoyo en el proceso de revisión editorial. 


\section{BIBLIOGRAFÍA}

Berger, A. (2014). Media and Communication Research Methods. An Introduction to Qualitative and Quantitative Approaches. Los Angeles: Sage.

Blair, H. (2013). Donors, Democratisation and Civil Society: Relating Theory to Practice. En Hulme, D. e Edwards, M. (eds.) NGOs, States and Donors. Too Close For Comfort? (23-42) Londres: Palgrave Macmillan.

Broom, G.M. y Sha, B.L. (2013). Cutlip and Center's Effective Public Relations (11a ed.). Harlow: Pearson.

Cano, M. (2014). Estratégias de Advocacy no Terceiro Setor enquanto função das Relações Públicas: campanhas de Advocacy no IMVF: um estudo de caso (Tesis de máster no publicada). Escola Superior de Comunicação Social - Instituto Politécnico de Lisboa, Portugal.

Coombs, W.T., y Holladay, S.J. (2014). It's Not Just PR: Public Relations in Society (2a ed.). Malden: Wiley Blackwell.

Creswell, J. W. (2014). Research design: Qualitative, quantitative, and mixed method approaches (3a Edición). Los Angeles: Sage.

Daly, J. (1998). If Communication Matters So Much, Why Don't We Get the Attention We Deserve? En J. S. Trent (eds.). Communication - Views from the Helm for the 21st Century (204 - 209). Needham Heights: Allyn y Bacon.

Eiró-Gomes, M. (2005). NGOs in Portugal: some remarks concerning organizational/corporate identity. En EUPRERA Proceedings.

Eiró-Gomes, M. (2006). Relações Públicas ou a Comunicação como acção. En Lição para o concurso de Professora Coordenadora, apresentado em Lisboa a 28 de Junho de 2006. Lisboa: Escola Superior de Comunicação Social.

Eiró-GOMES, M. y Lourenço, S. (2009). O papel e a responsabilidade das Relações Públicas na sustentabilidade de um mundo global. En Conferências Lusófona, 8o LUSOCOM, Lisboa, 1488-1499.

Eiró-Gomes, M., y Nunes, T. (2012). 3rd sector PR or when Community is our main stakeholder. Sinergie, rivista di studi e recherché, 89, 167-182.

Grunig, J. E. (1997). A situational theory of publics: Conceptual history, recent challenges and new research. En D. Moss, T. MacManus, \& D. Vercic (eds.). Public relations research: An International Perspective (3-46). London: International Thomson Business Press.

Machado, T. (2009). ONGD: O Papel da Comunicação no seu Conhecimento e Reconhecimento (Tesis de máster no publicada). Escola Superior de Comunicação Social. Instituto Politécnico de Lisboa, Portugal.

Mishra, V.K. (2012). The Role of Global Civil Society in Global Governance. Beijing Law Review, 3, 206-212. 
Montenegro, C. (2012). Comunicação para a saúde e para o desenvolvimento: Instituto Marquês de Valle Flôr (Tesis de máster no publicada). Escola Superior de Comunicação Social. Instituto Politécnico de Lisboa, Portugal.

Nunes, T. (2011). Terceiro Sector: Relações Públicas como Negociação e Compromisso (Tesis de máster no publicada). Escola Superior de Comunicação Social. Instituto Politécnico de Lisboa, Portugal.

Nunes, T. y Eiró-Gomes, M. (2013). Relações Públicas/ Comunicação Institucional/Comunicação Corporativa: três designações para uma mesma realidade?. En Livro de Atas $8^{\circ}$ Congresso SOPCOM: Comunicação Global, Cultura e Tecnologia, 1033-1040.

Pereira, M. (2015). Criação de uma Estratégia de Comunicação Integrada para uma Organização de Carácter Confessional (Tesis de máster no publicada). Escola Superior de Comunicação Social - Instituto Politécnico de Lisboa, Portugal.

Patterson, S. y Radtke, J. (2009). Strategic Communications for Nonprofit Organizations. Seven Steps to Creating a Successful Plan (2a Edición). Hoboken: John Wiley \& Sons.

Riddell, R. y Bebbington, A. (2013). Heavy Hands, Hidden Hands, Holding Hands? Donors, Intermediary NGOs and Civil Society Organisations. En D. Hulme y M. Edwards (eds.). NGOs, States and Donors - Too Close For Comfort? (107-127). Nova Iorque: Palgrave Macmillan.

Santos, M. (2015). Projecto de parceria entre a ONGD Estímulo e a Rede de Farmácias Portuguesas: a união de esforços entre sectores para a resolução de problemas contemporâneos (Tesis de máster no publicada). Escola Superior de Comunicação Social - Instituto Politécnico de Lisboa, Portugal.

Sodré, M. (2014). A ciência do comum: notas sobre o método comunicacional (1a Edición). Petropólis/RJ: Vozes.

Sousa, A. (2013). Campanha de comunicação - violência no trabalho: campanha de comunicação para a Associação Portuguesa de Apoio à Vítima (Tesis de máster no publicada). Escola Superior de Comunicação Social. Instituto Politécnico de Lisboa, Portugal.

UNDP (2012). UNDP Strategy on Civil Society and Civic Engagement. Disponible en http://www.undp.org/content/dam/undp/documents/partners/civil_society/publications/2012_UNDP_Strategy-on-Civil-Society-and-Civic-Engagement_EN_final.pdf

White, J., y Mazur, L. (1995). Strategic Communications Management. Singapore: Addison- Wesley Publishing Company.

Wilcox, D.L., Cameron, G.T. y Xifra, J. (2012). Relaciones Públicas: Estrategias y tácticas (10ma Edición). Madrid: Pearson.

Lei n. 66/98 de 14 de Outubro de 1998. Diário da República: I série. Disponible en www.dre.pt 